\title{
Human Identification by Measuring Respiration Patterns Using Vital FMCW Radar
}

\author{
Sangdong $\operatorname{Kim}^{1,2, *} \cdot$ Bongseok $\operatorname{Kim}^{1} \cdot$ Youngseok $\operatorname{Jin}^{1} \cdot$ Jonghun Lee $^{1,2}$
}

\begin{abstract}
This letter proposes a method of human identification that measures respiration patterns using frequency-modulated continuous wave (FMCW) radar. We exploit the fact that respiration signal patterns are unique to each individual, and FMCW radar is employed to obtain the respiration information. Based on the strengths of FMCW radar, the proposed algorithm compensates for the inability to distinguish the respiration signals of multiple users, which are difficult for continuous wave radar to measure. The proposed algorithm also employs a deep neural network algorithm instead of the K-nearest neighbor algorithm that was used in a previous study. The proposed algorithm further improves the performance by using a least mean square filter in the input signal of the DNN. The experimental results show that the proposed human identification method successfully classified four persons.
\end{abstract}

Key Words: Human Identification, Non-contact Radar, DNN, Vital FMCW Radar.

\section{INTRODUCTION}

Interest in non-contact sensor-based vital-sign detection has been growing, and several studies have been published that show that each individual has a unique pattern of breathing signals $[1$, 2]. Based on these results, it has been shown that user identification is possible using a pattern of vital signals [2]. Radar sensors have attracted particular attention as they are non-contact sensors, providing convenience to the user, and use radio waves, so they are resistant to external environmental factors, such as strong light, darkness, and fog. Radar systems can also continuously authenticate users [2].

In [2], the authors tried to identify users using a continuous wave $(\mathrm{CW})$ radar and a $\mathrm{K}$-nearest neighbor $(\mathrm{KNN})$ machine learning algorithm. However, CW radar employs only the fre- quency domain, making it difficult to distinguish the vital signals of multiple users, and the classification accuracy of the $\mathrm{KNN}$ algorithm can be degraded when the feature vector is not properly set.

This letter details two developments to improve the classification accuracy of radar-based authentication: first, a frequencymodulated continuous wave (FMCW) radar-based simultaneous recognition method, which has the advantage over $\mathrm{CW}$ radar of using both range and vital Doppler frequency parameters [3, 4], and second, a deep learning algorithm with enhanced performance for vital sign-based user authentication. A deep neural network (DNN) is suitable for the detection of vital signs with waveforms that change over time, and we therefore show in this letter improvement in the accuracy of user identification using the proposed algorithm.

\footnotetext{
Manuscript received April 14, 2020 ; Revised July 17, 2020 ; Accepted August 11, 2020. (ID No. 20200414-047J)

${ }^{1}$ Division of Automotive Technology, Daegu Gyeongbuk Institute of Science \& Technology, Daegu, Korea.

${ }^{2}$ Department of Interdisciplinary Engineering, Daegu Gyeongbuk Institute of Science \& Technology, Daegu, Korea.

"Corresponding Author: Sangdong Kim (e-mail: kimsd728@dgist.ac.kr)

Sangdong Kim and Bongseok Kim contributed equally to this work.
}

This is an Open-Access article distributed under the terms of the Creative Commons Attribution Non-Commercial License (http://creativecommons.org/licenses/by-nc/4.0) which permits unrestricted non-commercial use, distribution, and reproduction in any medium, provided the original work is properly cited.

(c) Copyright The Korean Institute of Electromagnetic Engineering and Science. All Rights Reserved. 


\section{SYSTEM MODEL AND PROPOSED ALGORITHM}

This section describes the system model and the proposed algorithm of the vital FMCW radar. Let a total of $L$ chirp signals be transmitted, reflected by $\mathrm{M}$ targets, and received. The received signal is dechirped by mixing the conjugate of the TX signal, and the dechirped signal is sampled as analog-to-digital converter (ADC). The $l$-th sampled beat signal is denoted by $y[n]$ for $0 \leq$ $n \leq N_{s}-1$, where the number of samples is $N_{s}$. By omitting the noise term, $y_{[}[n]$ is expressed as

$$
y_{l}[n]=\sum_{m=0}^{M-1}[\underbrace{a_{m} \underbrace{\exp \left(j 2 \pi \mu \tau_{m} n T_{s}\right)}_{\text {distance }} \times}_{\text {vital Doppler }} \times
$$

where $a_{m}$ is the complex amplitude of the $m$-th target, $\mu$ is the frequency slope of the FMCW chirp symbol, $\tau_{m}$ is the delay term due to the distance, $T_{s}$ is the sampling interval, $T$ is the duration of a chirp symbol, and $x_{m}^{(h)}$ and $x_{m, p}^{(r)}$ represent the $m$-th human's body motion by heartbeat and $p$-th harmonic term of respiration, respectively [5]. The sampled beat signal $y[n]$ is a column vector of size $N_{s}$ for $1 \leq l \leq L$ and is represented as a matrix $Y$ :

$$
\boldsymbol{Y}=\left[y_{1}[n], y_{2}[n], \ldots, y_{L}[n]\right] .
$$

The beat signal $\boldsymbol{Y}$ is the input to the human identification method using a respiration pattern; this method is performed as follows:

The proposed algorithm finds the distance parameter of multiple targets by using the fast Fourier transform results of the received beat signal [5]; high-classification accuracy is then obtained by performing the DNN using the vital Doppler signal of the distance index. When the heart rates of multiple targets are different, each target has a different vital Doppler component from the perspective of the FMCW signal. To classify targets based on each vital Doppler signal, various classification methods are usually employed. Of these, a KNN classifier is used for conventional identification systems [2], which is a kind of su-

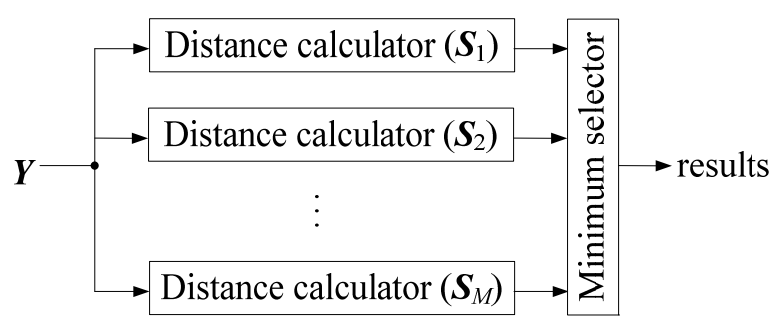

Fig. 1. Block diagram of KNN.

pervised learning that uses the labeled data to classify by referring to close labels of $k$ different data, as shown in Fig. 1. The KNN algorithm is a simple and effective method for multi-class classification and can model non-linear distributions. The KNN classifier uses a Euclidean distance method to calculate the distance between the $i$-th feature $S_{i}$ obtained from the training data and the features obtained from the testing data. The KNN classifier chooses $k$ training data, and the input feature is then assigned based on the smallest distance difference. However, the KNN algorithm is part of the machine learning category, and machine learning has lower accuracy than deep learning algorithms, such as the DNN algorithm upon which this paper focuses. Compared to other conventional machine learning algorithms, the DNN employs a multi-layer structure to improve generalization and abstraction performance.

For DNN training and testing, the vital Doppler signal from the received data is used to construct the complete dataset. In Fig. 2, the received vital Doppler signal is fed into the least mean square filter, which achieves signal-to-noise ratio improvement. The filtered signal matrix $R$ is the result of the adaptive filter. The output of the DNN is denoted by $H$ and is represented as

$$
\boldsymbol{H}=\sigma\left(\boldsymbol{W}_{k}^{\mathrm{T}} \cdots \sigma\left(\boldsymbol{W}_{2}^{\mathrm{T}} \sigma\left(\boldsymbol{W}_{1}^{\mathrm{T}} \boldsymbol{R}\right)\right)\right)
$$

where $W_{k}$ is the $k$-th weight matrix $k=1,2, \ldots, K ;(\cdot)^{\mathrm{T}}$ is a transpose operator; and $\sigma(\cdot)$ defines the activation function, such as ReLU.

The DNN with three hidden layers is selected as a network structure with final Softmax activation, as shown in Fig. 2. The activation function selected in the hidden layers is ReLU. By

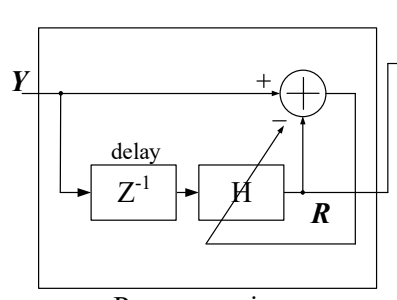

Pre-processing using LMS filter

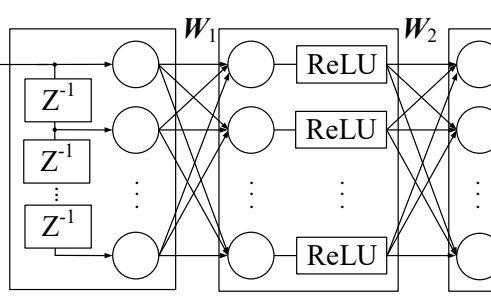

Input Layer
Hidden Layer 1

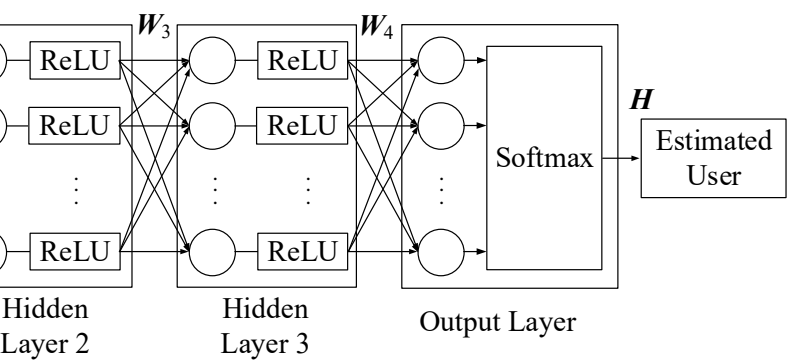

Fig. 2. Structure of proposed DNN. 


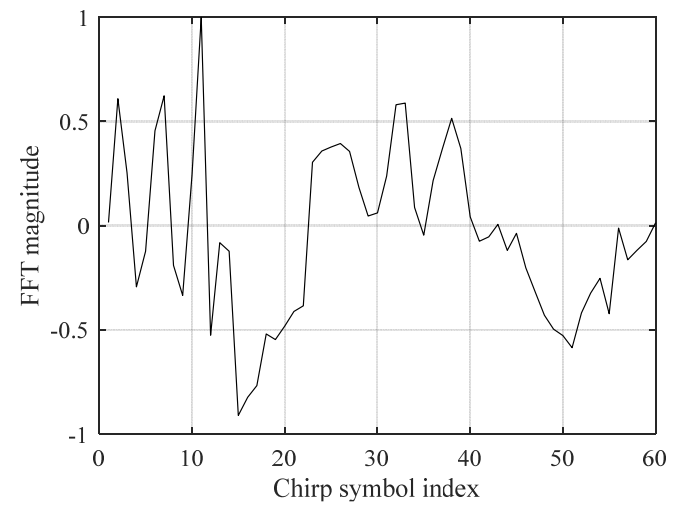

(a)

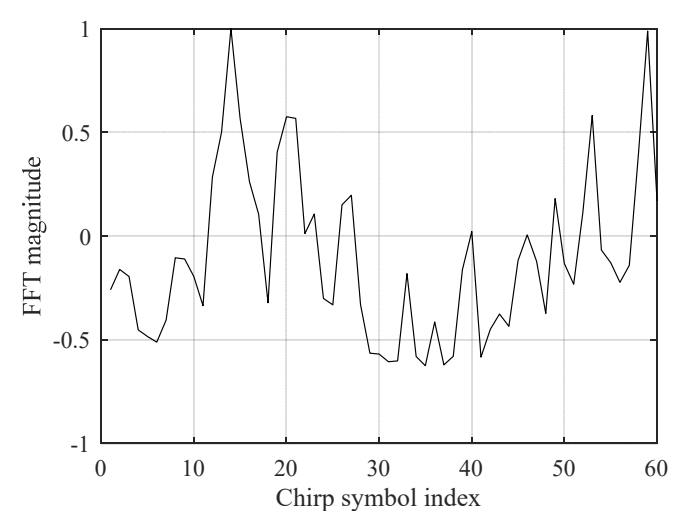

(c)

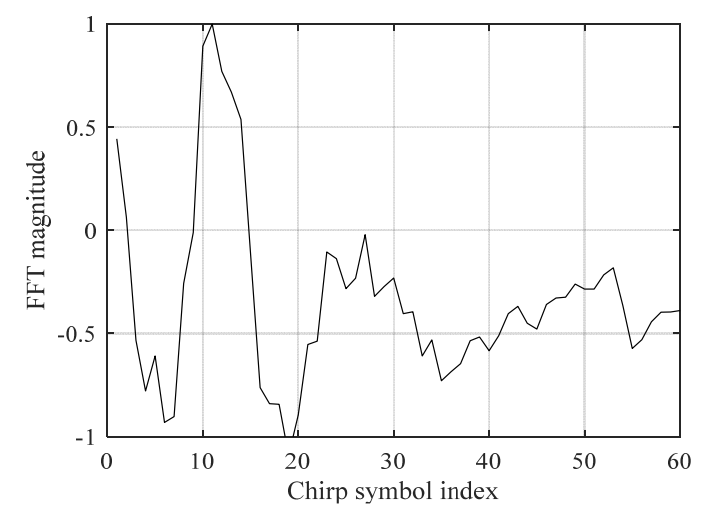

(b)

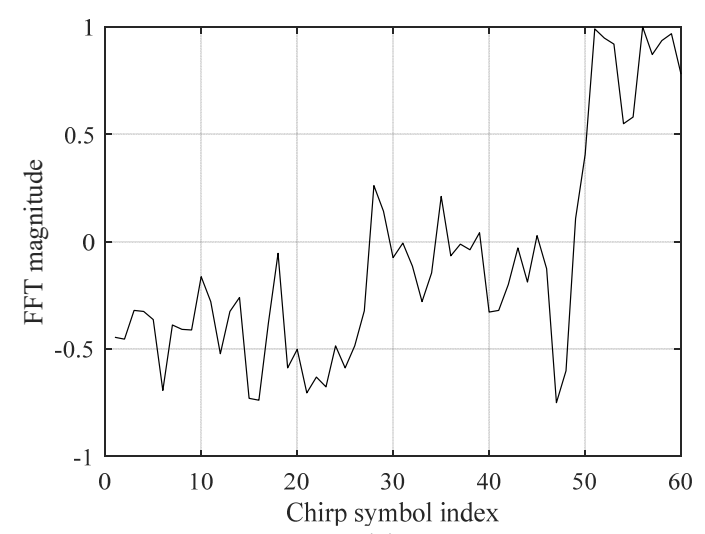

(d)

Fig. 3. Doppler signals of four subjects: (a) 1st person, (b) 2nd person, (c) 3rd person, and (d) 4th person.

using three hidden layers, it is possible to represent an arbitrary decision boundary with a rational activation function that can approximate any type of smooth mapping to any degree of accuracy, and additional layers can learn more complex representations. However, there is a tradeoff between architecture layers (deepness) and the computational complexity of the proposed system. In this work, significant accuracy improvements were not achieved by increasing the number of layers $(K>3)$, whereas the training process time was longer.

\section{EXPERIMENTS}

This section presents experimental results to verify the improvement of the proposed algorithm. An experiment was conducted in an indoor room in DGIST in South Korea. The overall experimental environment is shown in the simulation section.

We utilized the $24 \mathrm{GHz}$ FMCW radar system in [6]. The experiment sought the vital Doppler signal of the target selected from among the range estimation results from the human targets. The four human subjects described in this letter produced different vital Doppler signals, and these are shown in Fig. 3. For this experiment, various subjects' respiration and heartbeat signals, obtained for user authentication, were acquired.

Since the field of view for the radar system is within $\pm 15^{\circ}$ in [6], the number of people who can be measured simultaneously is two. The distance between the people in each experiment was therefore set to $2 \mathrm{~m}$. The experimental results are presented to demonstrate the improvement of the proposed algorithm. The four Doppler signals of the individuals are shown in Fig. 3. Human subjects have various vital Doppler signals, as shown in Fig. 3.

Table 1 shows the classifications of the KNN-based and proposed algorithms, comparing their performance. The input feature was of size $1 \times 60$ and $k$ was set to 3 , but the KNN-based user identification results are worse than those of the DNN-based algorithm.

The DNN used to classify the users consisted of an input layer of size $1 \times 60$, a 1 st hidden layer of size $60 \times 500$, a 2 nd hidden layer of size $500 \times 250$, a 3 rd hidden layer of size $250 \times$

Table 1. Classification accuracy of KNN-based and proposed algorithms

\begin{tabular}{ccccc}
\hline & \multicolumn{4}{c}{ Accuracy (\%) } \\
\cline { 2 - 5 } & User 1 & User 2 & User 3 & User 4 \\
\hline User 1 & $70 / 100$ & $0 / 0$ & $30 / 0$ & $0 / 0$ \\
User 2 & $10 / 0$ & $90 / 100$ & $0 / 0$ & $0 / 0$ \\
User 3 & $0 / 0$ & $0 / 0$ & $90 / 100$ & $10 / 0$ \\
User 4 & $0 / 0$ & $0 / 0$ & $0 / 0$ & $100 / 100$ \\
\hline
\end{tabular}

Values are presented as KNN/proposed algorithms. 
350, and an output layer of size $350 \times 4$. The DNN-based user identification results are better than those of the $\mathrm{KNN}$-based algorithm, as shown in Table 1, because the conventional algorithm using $\mathrm{KNN}$ had $95 \%$ accuracy.

\section{CONCLUSION}

This letter proposed a method of human identification by measuring respiration patterns using radar. The radar system overcomes the drawbacks of identification using specific body parts. Using KNN algorithms to classify multiple users has relatively poor classification results, and so a DNN algorithm was proposed to classify the respiration patterns of each person. The DNN-based user identification radar system was shown to classify four persons with $100 \%$ accuracy. In future research, heartbeat and respiration-based vital-sign data for user identification will be accumulated to improve vital monitoring techniques.

This work was supported by Daegu Gyeongbuk Institute of Science \& Technology (DGIST) and in part by the DGIST R\&D Program of the Ministry of Science, ICT, and Future Planning, Korea (No. 20-IT-02).

\section{REFERENCES}

[1] D. F. Proctor and J. B. Hardy, "Studies of respiratory air flow;

\section{Sangdong Kim}

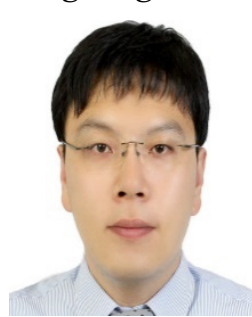

was born in Seoul, South Korea, in 1981. He received B.S. degree in Electronics Engineering from Hanyang University, Ansan, in 2004, M.S. degree in Electronics Engineering from Hanyang University, Seoul, in 2006 and Ph.D. degree in Electronics Engineering from Kyungpook National University, Daegu, in 2018. From 2015 to 2016, he was a visiting scholar at the University of Florida. From 2006, he has been working as a Senior Researcher with the Division of Automotive Technology, Daegu Gyeongbuk Institute of Science \& Technology (DGIST), Daegu, South Korea. And, from 2020, he has been working as an Assistant professor with the Department of Interdisciplinary Engineering (Adj.), Daegu Gyeongbuk Institute of Science \& Technology (DGIST). His research interests include super-resolution algorithms, automotive radar, and vital radar. significance of the normal pneumotachogram," Bulletin of the Johns Hopkins Hospital, vol. 85, no. 4, pp. 253-280, 1949.

[2] A. Rahman, V. M. Lubecke, O. Boric-Lubecke, J. H. Prins, and T. Sakamoto, "Doppler radar techniques for accurate respiration characterization and subject identification," IEEE Journal on Emerging and Selected Topics in Circuits and Systems, vol. 8, no. 2, pp. 350-359, 2018.

[3] D. Oh and J. H. Lee, "Low-complexity range-azimuth FMCW radar sensor using joint angle and delay estimation without SVD and EVD," IEEE Sensors Journal, vol. 15, no. 9, pp. 4799-4811, 2015.

[4] G. Wang, J. M. Munoz-Ferreras, C. Gu, C. Li, and R. GomezGarcia, "Application of linear-frequency-modulated continuous-wave (LFMCW) radars for tracking of vital signs," IEEE Transactions on Microwave Theory and Techniques, vol. 62, no. 6, pp. 1387-1399, 2014.

[5] S. Kim and K. K. Lee, "Low-complexity joint extrapolationMUSIC-based 2-D parameter estimator for vital FMCW radar," IEEE Sensors Journal, vol. 19, no. 6, pp. 2205-2216, 2019.

[6] S. Kim, D. Oh, and J. Lee, "Joint DFT-ESPRIT estimation for TOA and DOA in vehicle FMCW radars," IEEE Antennas and Wireless Propagation Letters, vol. 14, pp. 1710-1713, 2015.

Bongseok Kim

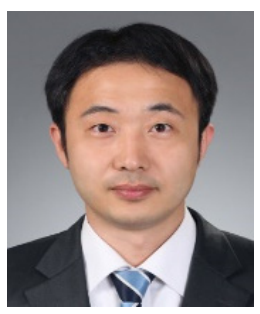

received his B.S. degree in Electronics Engineering in 2006 and M.S. and Ph.D. degrees in Information and Communications Engineering from Yeungnam University, Korea, in 2009 and 2014, respectively. From 2014 to 2016, he worked in Daegu Gyeongbuk Institute of Science \& Technology (DGIST), Korea, as a post-doc. Since 2016, he has been working in DGIST, Korea, as a senior research engineer. His current interests include multi-functional radar systems and radar signal processing. 


\section{Youngseok Jin}

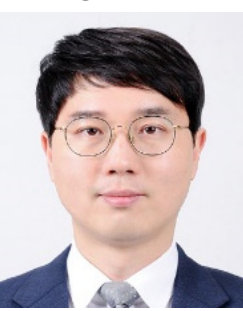

received B.S. and M.S. degrees in Communication Engineering from Daegu University, Korea, in 2010 and 2012, respectively. Since 2012, he has been working in Daegu Gyeongbuk Institute of Science \& Technology (DGIST), Korea, as a researcher. His current interests include radar signal processing and implementation in FPGA/DSP.

\section{Jonghun Lee}

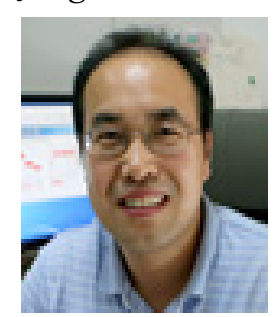

received B.S. degree in Electronics Engineering from Sungkyunkwan University, Korea, in 1996 and M.S. and Ph.D. degrees in Electrical and Electronics and Computer Science from Sungkyunkwan University, Korea, in 1998 and 2002, respectively. From 2002 to 2005, he worked in Samsung Electronics Company as a principal research engineer. Since 2005 , he has been working in the Division of Automotive Technology, Daegu Gyeongbuk Institute of Science \& Technology (DGIST), Korea, as a director \& principal researcher. And, he has been working as a full professor with the Department of Interdisciplinary Engineering of the DGIST. He is a IEEE senior member. His primary research interests are detection, tracking, recognition for radar (FMCW \& UWB radar), radar based vehicle sensor and radar signal processing and ML/DL for sensor fusion. 